\title{
Regulação emocional, bem-estar psicológico e bem-estar subjetivo
}

Vitor Santos Santana. Universidade Federal da Bahia.

Sônia Maria Guedes Gondim. Universidade Federal da Bahia.

\section{Resumo}

O artigo analisa as relações entre modos de regulação emocional e três dimensões de bem-estar subjetivo, a partir de dois modelos: um de predição e outro de mediação, que envolve efeitos indiretos da autonomia e do domínio do ambiente. Foram utilizadas três escalas, uma para avaliar dois modos de regulação emocional (up regulation e down regulation), outra para avaliar três dimensões de bem-estar subjetivo (afetos positivos, afetos negativos, satisfação com a vida) e outra para avaliar dois componentes do bem-estar psicológico (autonomia, domínio do ambiente). Também se incluiu um questionário sociodemográfico para caracterização da amostra. Participaram da pesquisa 231 brasileiros. Os resultados indicaram que os modos de regulação emocional foram capazes de prever os níveis de autonomia e de domínio do ambiente. O modelo de mediação indicou que a autonomia sozinha, foi capaz de mediar a relação entre os modos de regulação emocional e as dimensões de bem-estar subjetivo.

Palavras-chave: regulação emocional; bem-estar subjetivo; bem-estar psicológico; dimensões; mediação.

\begin{abstract}
Emotion regulation, psychological well-being and subjective well-being. This paper analyzes the relationship between emotion regulation modes and three dimensions of subjective well-being, from two models: a prediction model and another mediation involving the indirect effects of autonomy and environment mastery. The instrument of the study included three scales, one to evaluate two methods of emotional regulation (up regulation and down regulation), another to evaluate three dimensions of subjective well-being (positive affect, negative affect, life satisfaction) and another to assess two components of psychological well-being (autonomy, environmental mastery). Also included in the survey questions to characterize the sample. Participated in the survey 231 Brazilians. The results indicated that the modes of emotional regulation were able to predict the levels of autonomy and environment mastery. The mediation model indicated that the autonomy alone was capable of mediating the relationship between modes of emotion regulation and dimensions of subjective well-being.
\end{abstract}

Keywords: emotion regulation; subjective well-being; psychological well-being; dimensions; mediation.

\section{Resumen}

Regulación emocional, bienestar psicológico y bienestar subjetivo. En este artículo se analiza la relación entre los modos de regulación emocional y dimensiones de bienestar subjetivo, a partir de dos modelos: uno para predecir y otro de mediación que implica los efectos indirectos de la autonomía y el dominio del entorno. Se utilizaron tres escalas, una para evaluar dos modos de regulación emocional, otra para evaluar dimensiones del bienestar subjetivo y una otra para evaluar dos componentes del bienestar psicológico (autonomía y dominio del entorno). También se incluye en las preguntas de la encuesta para caracterizar la muestra. Los participantes del estudio fueron 231 brasileños. Los resultados indicaron que los modos de regulación emocional fueron capaces de predecir los niveles de autonomía y el dominio del entorno. El modelo de mediación indicó que la autonomía por sí solo fue capaz de mediar la relación entre los modos de regulación emocional y las dimensiones del bienestar subjetivo.

Palabras clave: regulación emocional; bienestar subjetivo; bienestar psicológico; dimensiones; mediación. 
As emoções são extremamente importantes na vida das pessoas e auxiliam na escolha de respostas adaptativas para enfrentar as dificuldades da vida cotidiana, além de preservar os laços sociais e o bem-estar pessoal (Lazarus, 1991). O processo de escolha que envolve compreender, equilibrar e decidir que emoções sentir e expressar é denominado de regulação emocional (Gross,1998a, 1999). Para Gross (2013), Koole (2009) e Tamir (2011), houve um crescimento expressivo da pesquisa sobre regulação na última década, passando a ser vista como um processo crucial na adaptação (Butler, Lee, \& Gross, 2007; Gross, 2002). Atos de regulação consciente demandam que a pessoa identifique seus estados afetivos desejáveis e se engaje em algum tipo de ação, pensamento ou comportamento para lidar, de forma adaptativa, com a experiência emocional momentânea (Carver, 2004; Cervone, Mor, Orom, Shadel, $\&$ Scott, 2004).

As evidências empíricas acumuladas sugerem haver formas mais eficazes de regulação, em termos das consequências afetivas e do bem-estar (Gross, 1998b; John \& Gross, 2004), o que torna importante a realização de mais estudos sobre estas relações. Apesar de existirem pesquisas internacionais com foco nas relações entre regulação emocional e bem-estar (Livingstone \& Srivastava, 2012; Quoidbach, Berry, Hansenne, \& Mikolajczak, 2010), pouco ainda tem sido feito no Brasil.

O estudo relatado neste artigo buscou contribuir para a investigação das relações entre dois modos de regulação emocional (ascendente - up regulation - e descendente - down regulation) e o bem-estar subjetivo, testando também o papel mediador de duas dimensões de bem-estar psicológico - a autonomia e o domínio do ambiente - nestas relações. O modelo de Nelis, Quoidbach, Hansenne e Mikolajczak (2011), adotado neste estudo, apresenta dois modos básicos de regulação emocional - up regulation (regulação ascendente) e down regulation (regulação descendente). A up regulation visa aumentar ou manter emoções positivas em situações que elas são ativadas, ao passo que a down regulation visa diminuir a intensidade de emoções negativas, quando ativadas. A capacidade de regular as emoções positivas (up regulation) é de particular relevância para a adaptação e a saúde mental, pois fortalece a atenção, a cognição e a criatividade, aumentando o bem-estar (Fredrickson \& Losana, 2005), a qualidade das relações sociais (Lopes, Salovey, Côté, \& Beers, 2005) e o desempenho no trabalho (Quoidbach $\&$ Hansenne, 2009).
O segundo principal construto incluído neste estudo é o de bem-estar. O bem-estar é um conceito polissêmico (Averill \& More, 1993), mas existem duas principais abordagens: a perspectiva hedônica e a eudaimônica (Mauss, Tamir, Anderson, \& Savino, 2011; McMahan \& Estes, 2011; Ryan \& Deci, 2001). Na perspectiva hedônica encontra-se o bem-estar subjetivo que se refere às avaliações pessoais sobre o nível de satisfação com a vida (Albuquerque \& Tróccoli, 2004; Diener, Oishi, \& Lucas 2003; Diener, Suh, Lucas, \& Smith, 1999; Keyes, Shmotkin, \& Ryff, 2002). Tais avaliações incluem a análise pessoal sobre a frequência com que se experimentam emoções positivas e negativas no dia a dia (Siqueira \& Padovan, 2008).

O bem-estar psicológico, por sua vez, apoia-se na perspectiva eudaimônica e se encontra relacionado ao desenvolvimento das potencialidades humanas e à busca da autorrealização e propósito de vida (Delle Fave, Brdar, Freire, Vella-Broderick, \& Wissing, 2013). Refere-se ao desenvolvimento humano obtido pela superação dos desafios existenciais de vida, contemplando várias dimensões do funcionamento psíquico (Keyes et al., 2002; Ryff \& Keyes, 1995; Ryff \& Singer 2008).

Diferentemente da visão hedônica de bem-estar subjetivo, o conceito de bem-estar psicológico vai além do sentimento de felicidade e satisfação geral com a vida, associado à vivência de mais afetos positivos que negativos (Ryff, 1989; Ryff \& Keyes, 1995). O modelo estrutural do funcionamento psicológico positivo de Ryff e Singer (2008) se apoia em diversas teorias humanistas clássicas na psicologia e é constituído por seis componentes: (i) Autoaceitação (atitudes positivas para consigo mesmo), (ii) Relacionamento positivo com outras pessoas (capacidade de amar e manter amizades), (iii) Autonomia (lócus interno de avaliação e o uso de padrões internos de autoavaliação), (iv) Domínio do ambiente (capacidade de escolher, criar e manejar ambientes complexos), (v) Propósito de vida (intenções e de senso de direção perante a vida), e (vi) Crescimento pessoal (abertura a novas experiências, para vencer desafios que se apresentam em diferentes fases da vida).

Uma revisão realizada por Machado e Bandeira (2012) afirma que diversos estudos investigaram a relação do bem-estar psicológico com processos desenvolvimentais adaptativos e construtos que refletem dimensões positivas da saúde mental (Ryff \& Singer, 2008). O bem-estar psicológico associou-se positivamente com autoestima e moral (Ryff, 1989), satisfação com a vida e afetos positivos (Ryff, 1989), coping (Holland 
\& Holahan, 2003), habilidades sociais (Segrin \& Taylor, 2007), inteligência emocional (Queroz \& Neri, 2005), resiliência (Ryff, Keyes, \& Hughes, 2003) e metas intrínsecas de crescimento (Bauer \& McAdams, 2004). Evidências empíricas sugerem que as pessoas que regulam adequadamente suas emoções tendem a ter níveis mais elevados de bem-estar (Gross \& Jonh, 2003; Nelis et al. 2011; Nyklíček, Denollet, \& Vingerhoets, 2011; Tamir, Mitchell, \& Gross, 2008).

O estudo de Quoidbach et al. (2010) examinou o impacto de estratégias de regulação emocional positivas em dois componentes do bem-estar subjetivo: afetos positivos e satisfação com a vida. Os resultados mostram que quando as pessoas mantêm as emoções positivas, seus níveis de afetos positivos e de satisfação com a vida aumentam proporcionalmente. Os resultados desse mesmo estudo também sugerem que, quando essas estratégias não são utilizadas, a experiência de estados negativos tende a ser mais frequente, diminuindo o bem-estar. Com base nesses resultados foram formuladas três hipóteses para o estudo: H1) Up regulation e down regulation relacionam-se positivamente com afetos positivos, satisfação com a vida, autonomia e domínio do ambiente; $\mathrm{H} 2$ ) Up regulation e down regulation relacionam-se negativamente com afetos negativos; e H3) Up regulation e down regulation são preditores das dimensões de bem-estar subjetivo (afeto positivo, afeto negativo e satisfação com a vida) e de autonomia e domínio do ambiente.

Neste estudo também procurou-se investigar se o bem-estar psicológico mediava a relação entre modos de regulação emocional e bem-estar subjetivo. O interesse de testar o papel mediador de duas dimensões de bem-estar psicológico nestas relações justifica-se pela possibilidade de explorar mais detalhadamente de que modo dimensões de bem-estar focadas em aspectos cognitivos (autonomia e domínio do ambiente) contribuem para explicar estados afetivos gerais. Em síntese, embora os dois construtos se refiram a bem-estar, o definem de modo diferenciado. $O$ bem-estar subjetivo mede um estado momentâneo, ao passo que o bem-estar psicológico apoia-se em traços pessoais, que se apresentam mais estáveis no tempo. Conforme assinala Warr (2007), evidências empíricas têm demonstrado que os modelos teóricos que investigam as concepções de bem-estar têm-se tornado mais complexos. Em resumo, a compreensão cada vez mais aprofundada dos fenômenos associados ao bem-estar requer elucidar melhor como variáveis antecedentes, moderadoras e/ou mediadoras se inter-relacionam e que de alguma maneira, influenciam o bem-estar (Mendonça, Ferreira, Porto, \& Zanini, 2012).

Os estudos de Burns e Machin (2010), por exemplo, buscaram identificar os efeitos do bem-estar psicológico no bem-estar subjetivo. $O$ estudo concluiu que o bem-estar psicológico torna-se um fator importante na previsão do bem-estar subjetivo. Segundo esses mesmos autores, a importância do bem-estar psicológico está em fornecer uma direção incluindo mudança de atitude mais duradoura, diminuindo a reatividade emocional para causas externas à pessoa, e que afetam o bem-estar subjetivo. O estudo de McMahan e Renken (2011) investigou as associações e mediações entre o bem-estar psicológico, percepções de significado da vida e bem-estar subjetivo. Os resultados indicaram que as dimensões de bem-estar psicológico foram associadas positivamente, tanto às percepções de significado na vida, quanto ao bem-estar subjetivo. As conclusões do estudo sugerem que a experiência de sentido na vida (eudaimônica) seria um caminho pelo qual as dimensões do bem-estar psicológico estão associadas a altos níveis de bem-estar subjetivo.

Conforme já descrito, além do modelo preditivo, o estudo apresentado neste artigo testou o papel mediador de duas dimensões do bem-estar psicológico, relacionadas à regulação emocional: a autonomia e o domínio do ambiente. A primeira dimensão diz respeito à autonomia pessoal, relacionada ao lócus interno de avaliação, fundamental para os processos de autorregulação. A segunda dimensão se refere à crença na capacidade de escolher ou criar ambientes adequados às características pessoais, o que também é central nos processos regulatórios. A mediação dessas dimensões na relação entre modo de regulação de emoções positivas e negativas fortaleceria as evidências da importância do bem-estar psicológico no alcance de melhores indicadores de bem-estar subjetivo, mais do que dos modos de regulação. Em virtude deste interesse de pesquisa formulou-se a hipótese 4: A relação entre modos de regulação emocional e dimensões de bem-estar subjetivo é mediada por autonomia e domínio do ambiente.

\section{Método}

\section{Participantes}

Os dados do estudo foram extraídos de uma amostra não probabilística de 231 pessoas residentes na região Metropolitana de Salvador/BA. Com relação às 
características pessoais, houve uma maior participação de mulheres $(N=132 ; 57,1 \%)$ e de solteiros $(N=137$; $59,3 \%$ ). A faixa etária média foi de 32,1 anos ( $D P=8,54$ e amplitude de 18 a 64 anos) e 139 pessoas (64,5\%) possuem nível superior completo. Todos os participantes estavam trabalhando na ocasião da coleta de dados, sendo que $32 \%(N=74)$ estavam no emprego há menos de 1 ano. Um total de $73 \%$ dos 157 restantes estavam no emprego entre 1 e 6 anos. As principais áreas de atuação eram na administração pública $(N=56)$, saúde $(N=45)$, indústria $(N=42)$, educação $(N=27)$, comunicação e informação $(N=25)$ e no comércio $(N=11)$.

\section{Instrumentos}

Perfil de regulação emocional - versão reduzida. O Perfil de regulação emocional versão portuguesa reduzida da medida desenvolvida por Nelis et al. (2011) (ver Gondim et al., 2015) é uma medida composta por seis cenários, três situações em que se prevê serem ativadas emoções negativas (raiva, tristeza e medo) e três nos quais se espera que os participantes ativem emoções positivas (excitação, alegria e gratidão). Após a descrição de cada situação são apresentadas oito opções de resposta (ex:"Você pula de alegria e expressa o seu entusiasmo") para lidar com a emoção desencadeada. Nos três cenários positivos, pode-se optar entre oito diferentes estratégias de regulação. Quatro são consideradas adaptativas (aumentam os efeitos benéficos das emoções positivas): 1) Manifestação do comportamento, que envolve a expressão de emoções positivas acompanhada de comportamentos não verbais sintonizados; 2 ) Saborear o momento presente, que se refere ao foco da atenção nas experiências momentâneas agradáveis; 3) Capitalização, que inclui comportamento como a comunicação e a celebração de eventos positivos com outros; e 4) Viagem mental positiva, que se refere ao resgate de lembranças positivas ou antecipação de eventos positivos. As outras quatro estratégias são consideradas desadaptativas (diminuem os efeitos benéficos das emoções positivas): 1) Inibição da expressão emocional, que envolve a supressão de emoções positivas; 2 ) Preocupação excessiva, que se refere à tendência a desviar o foco do evento positivo e a se preocupar constantemente com outras coisas; 3 ) Identificação de falhas, que envolve o foco em potenciais aspectos negativos da situação positiva ou no que poderia ser melhor; e 4) Viagem mental negativa, que se refere à ênfase na atribuição externa de eventos positivos e na antecipação negativa de consequências futuras.
Nos três cenários em que estão envolvidas emoções negativas, as quatro estratégias funcionais de regulação das emoções negativas (diminuem os efeitos danosos das emoções negativas) são: 1) Modificação da Situação, que envolve ação direta para transformar a situação e modificar seu impacto emocional; 2) Reorientação da atenção, que implica desvio do foco da atenção para modificar o sentimento; 3) Reavaliação positiva da situação, que se refere à mudança do pensamento sobre a situação inicialmente negativa; e 4) Expressão das emoções, que envolve o compartilhamento das emoções negativas com os outros. As quatro estratégias disfuncionais (aumentam os efeitos danosos da emoção negativa) são: 1) Desamparo aprendido, que se refere ao comportamento passivo acompanhado de um sentimento de impotência; 2) Ruminação, que envolve excesso de atenção a sentimentos e pensamentos associados a eventos negativos, aumentando a duração e intensidade das emoções negativas; 3) Abuso de substâncias, que envolve uso de bebidas alcoólicas, ansiolíticos ou drogas para evitar eventos adversos na tentativa de evitar mais consequências emocionais negativas; e 4) Reação impulsiva (Acting out), que se refere à ação irrefletida desencadeada por forte intensidade emocional.

Para cada tipo de regulação considerada adaptativa ou funcional escolhida é atribuído o valor 1,00; e para cada estratégia considerada desadaptativa ou disfuncional, o valor 1,00. O escore final representa o modo de regulação emocional ao lidar com as situações: predomínio da Up ou Down regulation. As cargas fatoriais encontradas neste estudo variaram de 0,73 a 0,78 , para as cinco situações, três que envolvem emoções positivas e duas que envolvem emoções negativas. Uma situação que envolve emoções negativas foi excluída, por apresentar carga fatorial abaixo de 0,25. O valor de alfa de Cronbach das situações após a análise fatorial variou de 0,74 a 0,76.

Escala de bem-estar psicológico. Baseada no modelo multidimensional proposto por Ryff (1989), a escala de bem-estar psicológico (EBEP) foi validada por Machado, Pawlowski e Bandeira (2013) para o português brasileiro, e desenvolvida para medir o bem-estar psicológico, por meio de seis componentes considerados importantes para o funcionamento humano saudável: auto-aceitação, relações positivas com os outros, crescimento pessoal, propósito na vida, o domínio com ambiente e autonomia (Keyes et al, 2002; Ryff \& Keyes, 1995). No presente estudo, foram utilizadas duas subescalas - autonomia (com cargas fatoriais variando de 0,75 a 0,78) e domínio do ambiente (com cargas fatoriais variando de 0,72 a 0,78 ), 
totalizando 12 itens. Os itens foram respondidos em uma escala tipo Likert de seis pontos, variando de (1) "discordo totalmente" a (6) "concordo totalmente". Os exemplos de itens são: "As pessoas dificilmente me convencem a fazer coisas que eu não queira" (Autonomia); "Eu sou muito bom em gerenciar as diversas responsabilidades da minha vida diária" (Domínio do ambiente). Os coeficientes alfa de Cronbach foram de 0,74 para autonomia e 0,77 para domínio do ambiente.

Escala de bem-estar subjetivo. Validada por Albuquerque e Tróccoli (2004) para o português brasileiro, a EBES possui um total de 62 itens distribuídos em três dimensões distintas: 21 itens para afetos positivos (ex: "agradável", $a=0,95) ; 23$ itens para afetos negativos (ex: "aflito", $a=0,95$ ) e 15 itens de satisfação com a vida (ex: "Avalio minha vida de forma positiva", $a=0,90$ ). Os itens foram respondidos em uma escala tipo Likert de seis pontos que varia de (1) "discordo totalmente" a (6) "concordo totalmente". Para o presente estudo, os coeficientes alfa de Cronbach foram de 0,84 para afetos positivos; 0,87 para afetos negativos e 0,88 para satisfação com a vida.

Além destas escalas algumas perguntas para caracterização dos participantes da amostra como sexo, idade, estado civil e nível educacional foram incluídas no survey.

\section{Procedimentos de Coleta de Dados}

Os dados foram coletados de duas formas: 1) Mediante questionário on-line, em que se fez uso do aplicativo EF Survey e 2) por questionário impresso, sendo a aplicação acompanhada pelo pesquisador. Os participantes foram recrutados mediante a divulgação em empresas e redes sociais. 102 pessoas (44,15\%) responderam o questionário no formato on-line, e 150 via questionário impresso, porém 129 (55,85\%) foram considerados válidos, por estarem completamente respondidos, totalizando 231 casos válidos. Para o teste de homogeneidade da amostra foi realizado o teste $t$ não sinalizando haver diferença significativa entre os métodos de coleta.

\section{Procedimentos para Análise de Dados}

Foram realizadas estatísticas descritivas de tendência central (média) e desvio-padrão (DP). Para descrever o relacionamento entre as variáveis (hipóteses $\mathrm{H} 1$ e H2) foram utilizadas correlações bivariadas ( $r$ de Pearson). As hipóteses H3 (modelo de predição) e H4 (modelo de mediação) foram testadas pela-técnica de modelagem de equações estruturais (MEE) (Arbuckle, 2010). Para este estudo, foi utilizada a matriz de covari- ância das variáveis e o método de estimação escolhido foi Maximum Likelihood (MLE). A existência de valores atípicos (outliers) multivariados foi avaliada pela distância quadrada de Mahalanobis $\left(\mathrm{DM}^{2}\right)$ e os pressupostos de normalidade das variáveis foram avaliados pelos coeficientes de assimetria (Sk) e curtose (Ku) (Marôco, 2010).

Os indicadores de ajustamento dos modelos usados no estudo foram: razão entre o qui-quadrado e os graus de liberdade do modelo $\left(\mathrm{x}^{2} / \mathrm{gl}\right)$ menor do que 5 (Byrne, 2001); o CFI (Comparative Fit Index) e o AGFI (Adjusted Goodness-of-Fit Index) maiores do que 0,90 (Hu \& Bentler, 1999); o RMSEA (Root Mean Square Error of Approximation) menor do que 0,05 Recomendam-se valores próximos a 0,06 e 0,08 e com seu intervalo de confiança de 90\% (IC 90\%); e o AIC (Akaike Information Criterion) para fins de comparação de modelos fatoriais em que o modelo que apresenta menor valor de AIC tem um melhor ajustamento aos dados, por ser mais parcimonioso (Marôco, 2010).

\section{Análises Preliminares do Modelo Hipotetizado}

Para a AFC (Análise Fatorial confirmatória) foram especificadas sete variáveis latentes: duas representando modos de regulação emocional (up e down regulation); duas representando o bem-estar psicológico (autonomia e domínio do ambiente) e três representando as dimensões de bem-estar subjetivo (afetos positivos, afetos negativos e satisfação com a vida). Cada variável latente possui três indicadores gerados pela combinação de itens a partir das recomendações de Coffman e MacCallun (2005).

De acordo com Byrne (2001), para garantir a identificação estatística dos modelos, em cada variável latente foi fixado o valor 1 . Os resultados mostram que os índices de qualidade de ajustamento do modelo hipotetizado foram: $\chi^{2}(131)=289,957 ; p<0,001 ; \chi^{2} / g . I=2,21 ; C F I=$ 0,$924 ; \mathrm{GFI}=0,93$ e RMSEA IC $90 \%=0,073(0,061-0,084)$, com cargas fatoriais variando de 0,42 a 0,92.

A fim de garantir a validade do modelo hipotetizado, esses resultados foram comparados a um modelo alternativo, em que todos os itens formavam um fator latente geral em cada dimensão. Os resultados deste modelo foram: $\chi^{2}(149)=969,065 ; p<0,001 ; \chi^{2} / g . l=6,05$; $\mathrm{CFI}=0,72 ; \mathrm{GFI}=0,62$ e RMSEA IC $90 \%=0,15(0,14-0,16)$, considerado um modelo de ajustamento pobre. A variação da estatística qui-quadrado entre os dois modelos foi de $\Delta \chi^{2}(18)=679,108, p<0,01$. Como o valor de $\Delta \chi^{2}$ foi maior e significativo, pode-se concluir que os resultados da AFC apoiam o modelo com sete fatores. 


\section{Resultados}

\section{Estatísticas Descritivas e Correlações}

Para o teste das hipóteses $\mathrm{H} 1$ e $\mathrm{H} 2$ foram realizadas estatísticas descritivas e correlações bivariadas entre as variáveis do estudo, como mostra a Tabela 1. A variável up regulation está relacionada positivamente com satisfação com a vida $(r=0,30)$, autonomia $(r=0,33)$ e domínio do ambiente $(r=0,29)$ todos com $p s<0,01$. Os afetos positivos apresentaram uma relação positiva com up regulation $(r=0,18 ; p<0,05)$ e com um efeito menor, down regulation $(r=0,10 ; p<0,05)$.

Observou-se um relacionamento positivo entre down regulation e satisfação com a vida $(r=0,21)$, autonomia $(r=0,32)$ e domínio do ambiente $(r=0,30)$, todos com $p<0,01$. A variável afetos negativos relacionou-se negativamente com up regulation $(r=0,26)$, porém não houve significância estatística desta variável no caso da down regulation. Autonomia e domínio do ambiente apresentaram relação positiva com a satisfação com a vida ( $r=0,64$ e $r=0,50$ todos com $p<0,01)$, respectivamente. Todas as correlações variaram de fracas a moderadas, indicando que as variáveis não apresentaram multicolinearidade.

\section{Modelo de Predição}

Com o objetivo de testar o poder preditivo das duas estratégias de regulação emocional foram inseridas as variáveis up regulation e down regulation como preditoras. Como variáveis critério, foram inseridas autonomia, domínio do ambiente, afetos positivos, afetos negativos e satisfação com a vida. Segundo Frazier, Tix e Barron (2004), antes de testar qualquer modelo de mediação é necessário checar o efeito das variáveis de predição nas demais variáveis inseridas no modelo. É ideal que haja uma relação significante entre os preditores e mediadores e entre os preditores e as variáveis critério. Dessa forma, esse modelo além de possuir a função de um modelo de predição, também atua como uma fase preliminar para a composição do modelo de mediação.

Como mostra a Figura 1, up regulation obteve um efeito significativo com afetos positivos $(\beta=0,15$; $p<0,001)$, satisfação com a vida $(\beta=0,26 ; p<0,001)$, autonomia $(\beta=0,24 ; p<0,001)$ e domínio do ambiente $(\beta=0,28 ; p<0,001)$. Down regulation também obteve um efeito significativo nas variáveis satisfação com a vida $(\beta=0,18 ; p<0,001)$, autonomia $(\beta=0,33 ; p<0,001)$ e domínio do ambiente $(\beta=0,31 ; p<0,001)$. Por outro lado, somente up regulation obteve um efeito preditivo significativo em afetos negativos $(\beta=0,27 ; p<0,001)$.

O Modelo 1, após a remoção das trajetórias não significativas, apresentou índices de ajustamento iguais a $\chi^{2}(177)=381,333 ; p<0,001 ; \chi^{2} / g . I=2,1 ; C F I=0,869$; $\mathrm{GFI}=0,898$ e RMSEA IC $90 \%=0,071(0,061-0,081)$, $A I C=489,33$. Juntas, as variáveis preditoras up e down regulation explicam as seguintes parcelas de variância $\left(R^{2}\right)$ das dimensões de bem-estar subjetivo: $13 \%$ de afetos positivos; $12 \%$ de afetos negativos, $32 \%$ de satisfação com a vida. Com efeito, a variância total explicada do construto foi de $57 \%$, corroborando com achados anteriores (Quoidbach et al., 2010).

Após a verificação dos efeitos preditivos pelo Modelo 1, constata-se neste estudo, que a variável up regulation é capaz de explicar um maior número de dimensões de bem-estar subjetivo do que a down regulation. Porém, os efeitos explicativos nas variáveis autonomia e domínio do ambiente são mais bem explicados pela variável down regulation. Como não houve um efeito significativo entre down regulation, afetos positivos $(\beta=0,03)$ e afetos negativos $(\beta=0,03)$ todos com $p>0,05$, essas trajetórias foram removidas para compor o Modelo 2.

Tabela 1. Estatísticas Descritivas e Correlações Bivariadas de Todas as Variáveis.

\begin{tabular}{|c|c|c|c|c|c|c|c|c|c|}
\hline Variáveis & Média & DP & 1 & 2 & 3 & 4 & 5 & 6 & 7 \\
\hline Up regulation & 2,65 & 0,68 & - & & & & & & \\
\hline Down regulation & 1,84 & 0,58 & $0,36^{* *}$ & - & & & & & \\
\hline Afetos Positivos & 3,35 & 0,82 & $0,18^{*}$ & $0,10^{*}$ & - & & & & \\
\hline Afetos Negativos & 2,38 & 0,84 & $-0,26^{* *}$ & $-0,12$ & $-0,35^{* *}$ & - & & & \\
\hline Satisfação com a vida & 4,07 & 0,71 & $0,30^{* *}$ & $0,21^{* *}$ & $0,55^{* *}$ & $-0,45^{* *}$ & - & & \\
\hline Autonomia & 4,66 & 0,85 & $0,33^{* *}$ & $0,32^{* *}$ & $0,40^{* *}$ & $-0,34^{* *}$ & $0,64^{* *}$ & - & \\
\hline Domínio do ambiente & 4,03 & 0,91 & $0,29^{* *}$ & $0,30^{* *}$ & $0,41^{* *}$ & $-0,31^{* *}$ & $0,50^{* *}$ & $0,57^{* *}$ & - \\
\hline
\end{tabular}




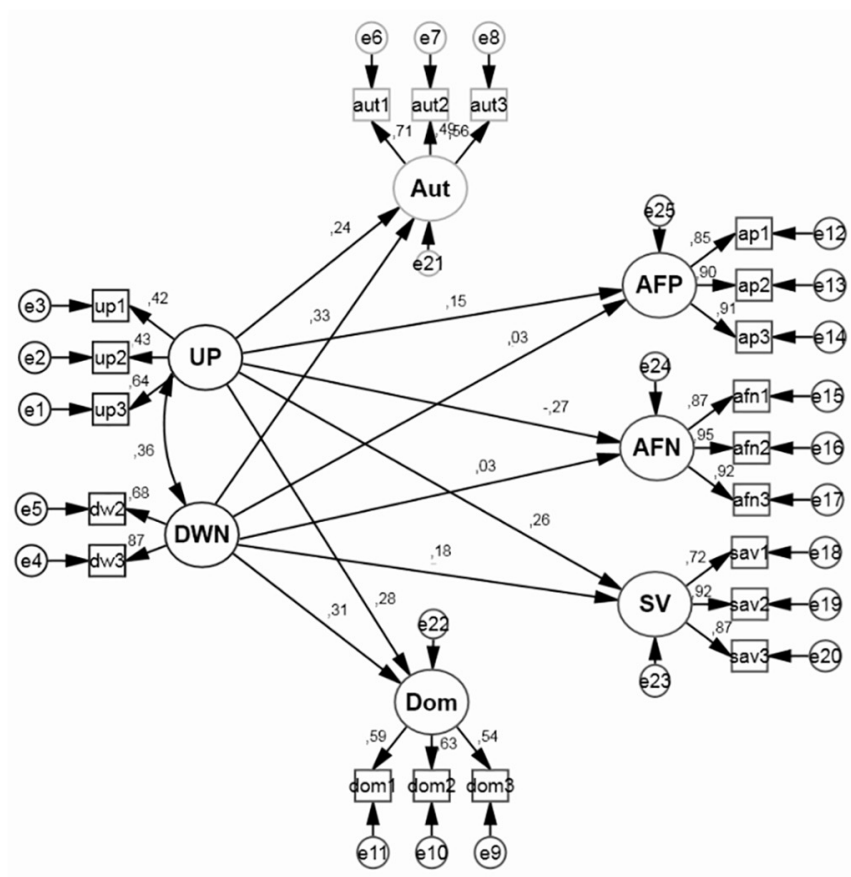

Figura 1. Modelo de Predição Entre as Estratégias de Regulação Emocional e Dimensões de Bem-Estar Psicológico e Subjetivo. UP: up regulation; DWN: down regulation; Aut: autonomia; Dom: domínio do ambiente; AFN: afetos negativos; AFP: afetos positivos; SV: satisfação com a vida.

\section{Modelo de Mediação}

O Modelo 2 teve como objetivo testar os efeitos mediadores de autonomia e domínio do ambiente, na relação entre as estratégias de regulação emocional e bem-estar subjetivo. Os efeitos totais de up $(\beta=0,13)$ e down regulation $(\beta=0,33)$ foram significativos $(p<0,01)$, indicando que altos níveis de up e down regulation produzem altos níveis de afetos positivos e satisfação com a vida. Os efeitos totais também indicaram que, quanto maiores níveis de regulação emocional, menores serão os níveis de afetos negativos. Esses resultados se assemelham aos estimados pelo Modelo 1.

Após a análise dos efeitos totais pode-se observar que na relação entre os preditores e os mediadores, down regulation apresentou coeficientes padronizados maiores que up regulation. De acordo com Preacher e Hayes (2004), os efeitos totais correspondem aos efeitos das variáveis preditoras antes da inserção dos mediadores no modelo. Assim, quanto maiores os níveis de down regulation, maiores serão os níveis de autonomia $(\beta=0,31 ; p<0,001)$ e domínio do ambiente $(\beta=0,19$; $p<0,001)$. Porém, na relação entre os mediadores e as variáveis critério, somente o efeito da autonomia foi significativo na explicação de afetos positivos $(\beta=$ $0,41 ; p<0,001)$, afetos negativos $(\beta=-0,54 ; p<0,001)$ e satisfação com a vida ( $\beta=0,75 ; p<0,001)$. Neste modelo, os efeitos diretos com a inserção dos mediadores não foram significativos e diferentes de zero, o que sugere uma mediação total (Baron \& Kenny, 1986) (Figura 2).

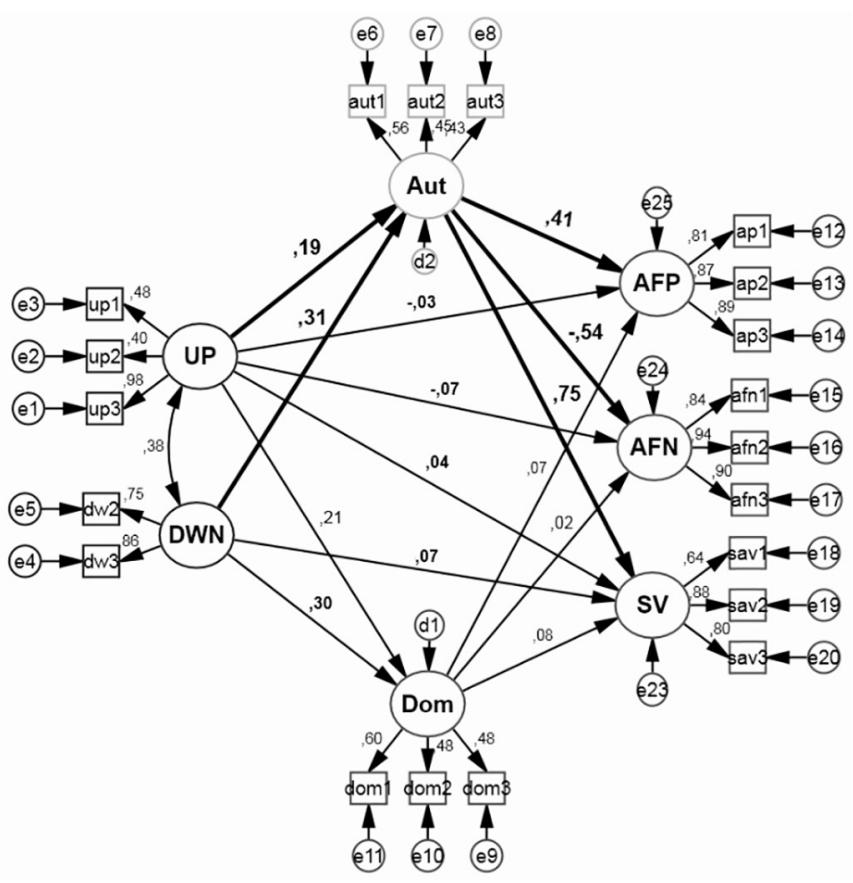

Figura 2. Modelo de Mediação pela Autonomia e Domínio do Ambiente na Relação entre as Estratégias de Regulação Emocional e Dimensões de Bem-Estar Subjetivo. UP: up regulation; DWN: down regulation; Aut: autonomia; Dom: domínio do ambiente; AFN: afetos negativos; AFP: afetos positivos; SV: satisfação com a vida.

Finalmente, para verificar a significância da mediação, os efeitos indiretos foram submetidos ao teste de Sobel (1982) e calculados por meio do macro de Preacher e Hayes (2004). Os efeitos indiretos de up regulation por meio da autonomia foram significativos para afetos positivos ( $\beta_{\text {indireto }}=0,10 ;$ Zsobel $=3,19 ; p<0,001$ ), afetos negativos $\left(\beta_{\text {indireto }}=0,10 ;\right.$ Zsobel $\left.=4,04 ; p<0,001\right)$ e satisfação com a vida ( $\beta_{\text {indireto }}=0,16$; Zsobel $=5,86 ; p$ $<0,001)$. A variável down regulation também obteve um resultado significativo por meio da autonomia para a variável satisfação com a vida $\left(\beta_{\text {indireto }}=0,26\right.$; Zsobel $=$ $0,26 ; p<0,001)$. Portanto, pode-se afirmar que neste modelo, somente a autonomia exerce papel mediador na relação entre as estratégias de regulação emocional e as dimensões de bem-estar subjetivo. Após a criação e análise dos modelos é possível realizar uma comparação pelos índices de qualidade de ajustamento, com o propósito de identificar o modelo que melhor explica as relações entre as variáveis do estudo (Marôco, 2010). Modelo de Predição: $\chi^{2}(177)=381,333 ; p<0,001 ; \chi^{2} / g$.I 
$=2,1 ; \mathrm{CFI}=0,869 ; \mathrm{GFI}=0,898$ e RMSEA IC 90\% $=0,071$ $(0,061-0,081)$, AIC $=489,33$, Modelo de mediação: $\chi^{2}$ $(177)=298,902 ; p<0,001 ; \chi^{2} / g . I=1,66 ; C F I=0,938$; $\mathrm{GFI}=0,903$ e RMSEA IC $90 \%=0,07(0,06-0,08), \mathrm{AIC}=$ 318,145 . Os valores do Modelo 2 (mediação) foram meIhores em comparação ao Modelo 1 (predição), sendo aquele, portanto, o que melhor se ajusta aos dados da amostra do estudo.

\section{Discussão}

Como previsto pela Hipótese $\mathrm{H} 1$, encontrou-se correlações positivas entre up e down regulation e as dimensões de afetos positivos e de satisfação com a vida, corroborando os resultados de pesquisas anteriores (Haga, Kraft, \& Corby, 2009; Nelis et al., 2011). Esses achados permitem inferir que quanto maiores são os níveis de regulação emocional, maiores serão as experiências emocionais afetivas e melhores avaliações sobre os aspectos globais da vida. Como previsto pela Hipótese $\mathrm{H} 2$, quanto maiores os níveis de regulação de emoções positivas (up regulation), menores serão os estados de engajamento afetivo negativo. Porém, essa associação não foi significante para regulação dos efeitos das emoções negativas (down regulation). Era esperado que essa relação fosse significante, de acordo com pesquisas anteriores (Gross, 2013; Nelis et al., 2011). A baixa associação entre a regulação dos efeitos das emoções negativas (down regulation), com os estados afetivos negativos e positivos, ocorreu porque no entendimento dos autores deste artigo esse modo de regulação emocional não obedece à mesma lógica que a regulação de emoções positivas. Quando o objetivo é reduzir emoções negativas, é necessário que a pessoa realize um esforço cognitivo maior, para que os estados afetivos positivos sejam mantidos e estados negativos sejam reduzidos, na mesma proporção.

Em contraste, manter emoções positivas parece ser uma condição mais favorável para ambas as dimensões de bem-estar. Alinhada à hipótese $\mathrm{H} 3$ relacionada à predição, ao optarem por manter as emoções positivas, as pessoas acabam por influenciar seus estados afetivos e suas avaliações gerais sobre a vida. Esses achados corroboram estudos recentes de Livingstone e Srivastava (2012) e de Quoidbach et al. (2010), onde se evidenciou que, ao se valerem de estratégias de regulação emocional para potencializar os efeitos das emoções positivas, as pessoas desejam melhorar seus níveis de bem-estar, o que provavelmente favorece a motivação para a busca do bem-estar.
Além de influenciarem as dimensões de bem-estar subjetivo, os modos de regulação foram capazes de prever os níveis de autonomia e de domínio do ambiente. As estratégias de regulação emocional, além de influenciarem a utilização de padrões internos para um funcionamento psicológico adequado, também contribuem na escolha ou criação de ambientes externos mais favoráveis às características pessoais. Por serem componentes cognitivos e que influenciam as avaliações pessoais (Ryan \& Deci, 2001), a autonomia e o domínio do ambiente sofrem efeito das estratégias emocionais, pois, ao manejar adequadamente uma emoção positiva ou negativa, as autoavaliações e as avaliações dos ambientes externos tendem a ser mais favoráveis.

Os resultados do modelo de mediação suportam parcialmente a hipótese $\mathrm{H} 4$, pois esta relação de mediação não foi confirmada pelos efeitos indiretos do domínio do ambiente. Com isso, evidencia-se que a autonomia sozinha é capaz de mediar a relação entre os efeitos dos modos de regulação emocional e as dimensões de bem-estar subjetivo. A relação de mediação corrobora os estudos de Burns e Machin (2010), indicando que o bem-estar psicológico é uma via para se alcançar bons níveis de bem-estar subjetivo. Para Ryan e Deci, (2001), uma pessoa que autenticamente endossa suas ações, faz escolhas a partir de si mesmo e reflete seus verdadeiros interesses e valores, está agindo de forma autônoma. Dessa forma, ao se submeterem a situações em que emoções positivas e negativas estão presentes, as pessoas que apresentam maiores níveis de autonomia, provavelmente, irão regular melhor estas emoções, quando o propósito é atingir metas de bem-estar subjetivo mais elevado.

Em síntese, os resultados do estudo confirmaram a hipótese de que os modos de regulação emocional de fato relacionam-se com as dimensões de bem-estar subjetivo e psicológico. Os achados deste estudo também indicam que quando a autonomia está presente na relação, maiores serão as chances das pessoas vivenciarem melhor bem-estar subjetivo. Nesse sentido, os resultados apresentados neste estudo convergem parcialmente com a literatura empírica sobre o tema sobre o papel importante que dimensões do bem-estar psicológico exercem no bem-estar subjetivo (felicidade). Os modelos de predição e de mediação se mostraram relevantes, ao analisar o papel exercido pelos modos de regulação emocional nos dois tipos de bem-estar, o psicológico e o subjetivo. Estes resultados também trazem evidências de que bem-estar psicológico e bem-estar subjetivo, apesar de terem afinidades conceituais, diferenciam-se 
na contribuição que oferecem à percepção de felicidade e satisfação geral com a vida. Estados afetivos momentâneos de bem-estar, portanto, podem ser potencializados por traços pessoais, como a autonomia. Ajudam também a compreender que a regulação emocional pode ter um efeito indireto sobre o bem-estar subjetivo pela via da autonomia. A importância disto é reconhecer que processos de socialização em diversos contextos (educacionais, de trabalho, etc.) e que estimulem a autonomia podem potencializar os efeitos da regulação emocional no bem-estar subjetivo.

Há que se considerar que os dados apresentados são correlacionais, e nada se pode inferir, com certeza, sobre a direção da influência causal entre as variáveis. Mesmo que seja possível que a regulação emocional afete as dimensões de bem-estar, uma relação contrária de reciprocidade pode ser esperada, isto é, o bem-estar influenciando a regulação emocional. Mais pesquisas utilizando um desenho experimental ou longitudinal são recomendáveis para determinar a direção de causalidade. Outras investigações podem explorar também as possíveis relações entre regulação emocional e as outras dimensões de bem-estar psicológico como a autoaceitação, relações positivas com outros, crescimento pessoal e propósito na vida, incluindo as dimensões de bem-estar subjetivo exploradas neste estudo.

\section{Referências}

Albuquerque, A. S., \& Tróccolli, T. B. (2004). Desenvolvimento de uma escala de bem-estar subjetivo. Psicologia: Teoria e Pesquisa, 20(2), 153-164. doi: 10.1590/S0102-37722004000200008

Arbuckle, J. L. (2010). IBM SPSS Amos 19 User's Guide. Chicago: IL. IBM.

Averill, J., \& More, T. (1993). Happiness. In M. Lewis \& J. Haviland (Orgs.), Handbook of emotions (pp. 617-629). Nova lorque: The Guilford Press.

Baron, R. M., \& Kenny, D. A. (1986). The moderator-mediator variable distinction in social psychological research: conceptual, strategic, and statistical considerations. Journal Personality and Social Psychology, 51(6), 1173-1182. doi: 10.1037/0022-3514.51.6.1173

Bauer, J. J., \& McAdams, D. P. (2004). Personal growth in adults' stories of life transitions. Journal of Personality, 72(3), 573-602. doi: 10.1111/j.0022-3506.2004.00273.x

Burns, R. A., \& Machin, M. A (2010). Identifying gender differences in the independent effects of personality and psychological wellbeing on two broad affect components of subjective well-being. Personality and Individual Differences, 48, 22-27. doi: 10.1016/j. paid.2009.08.007

Butler, E. A., Lee, T. L., \& Gross, J. J. (2007). Emotion regulation and culture: are the social consequences of emotion suppression culturespecific? Emotion, 7(1), 30-48. doi: 10.1037/1528-3542.7.1.30
Byrne, B. (2001). Structural equation modeling with Amos: basic concepts, applications and programming. Nova Jersey: Lawrence Erlbaum Associates.

Carver, C. S. (2004). Self-regulation of action and affect: Nova lorque: Guilford Press.

Cervone, D., Mor, N., Orom, H., Shadel, W. G., \& Scott, W. D. (2004). Self-efficacy beliefs on the architecture of personality: on knowledge, appraisal, and self-regulation. Nova lorque: Guilford Press.

Coffman, D. L., \& MacCallun, R. C. (2005). Using parcels to convert path analysis models into latent variable models. Multivariate Behavioral Research, 40(2), 235-259. doi: 10.1207/s15327906mbr4002_4

Delle Fave, A., Brdar, I., Freire, T., Vella-Brodrick, D., \& Wissing, M. (2011). The eudaimonic and hedonic components of happiness: qualitative and quantitative findings. Social Indicators Research, 100(2), 185207. doi: $10.1007 / \mathrm{s} 11205-010-9632-5$

Diener, E., Oishi, S., \& Lucas, R. E. (2003). Personality, culture, and subjective well-being: emotional and cognitive evaluations of life. Annual Review of Psychology, 54, 403-425. doi: 10.1146/annurev. psych.54.101601.145056

Diener, E., Suh, E. M., Lucas, R. E., \& Smith, H. L. (1999). Subjective well-being:three decades of progress. Psychological Bulletin, 125(2), 276-302. doi: 10.1037/0033-2909.125.2.276

Frazier, P. A., Tix, A. P., \& Barron, K. A. (2004). Testing moderator and mediator effects in counseling psychology. Journal of Counseling Psychology, 51, 115-134. doi: 10.1037/0022-0167.51.1.115

Fredrickson, B. L., \& Losada, M. F. (2005). Positive affect and the complex dynamics of human flourishing. American Psychologist, 60(7), 678-686. doi: 10.1037\%2F0003-066X.60.7.678

Gondim, S. M. G., Pereira, C., Hirschle, A. L. T., Palma, E. M. S., Alberton, G. D., Paranhos, J., ... Ribeiro, W. (2015). Evidências de validação de uma medida de características pessoais de regulação das emoções. Psicologia, Reflexão e Crítica, 28(4), 659-667. doi: 10.1590/16787153.201528403

Gross, J. J. (1998a). Antecedent and response-focused emotion regulation: divergent consequences for experience, expression, and physiology. Journal of Personality and Social Psychology, 74, 224-237. doi: 10.1037/0022-3514.74.1.224

Gross, J. J. (1998b). The emerging field of emotion regulation: an integrative review. Review of General Psychology, 2(3), 271-299. doi: 10.1037/1089-2680.2.3.271

Gross, J. J. (1999). Emotion regulation: past, present, future. Cognition and Emotion, 13(5), 551-573. doi: 10.1080/026999399379186

Gross, J. J. (2002). Emotion regulation: affective, cognitive, and social consequences. Psychophysiology, Cambridge University Press. 39, 281-291. doi: 10.1017.S0048577201393198

Gross, J. J. (2013). Emotion regulation: taking stock and moving forward. Emotion, 13(3), 359-365. doi: 10.1037/a0032135.

Gross, J. J., \& John, O. P. (2003). Individual differences in two emotion regulation processes: Implications for affect, relationships and wellbeing. Journal of Personality and Social Psychology, 85(2), 348-362. doi: 10.1037/0022-3514.85.2.348

Haga, S. M., Kraft, P., \& Corby, E. K. (2009). Emotion regulation: antecedents and well-being outcomes of cognitive reappraisal and expressive suppression in cross-cultural samples. Journal of Happiness, 10, 271-291. doi: 10.1007/s10902-007-9080-3 
Regulação emocional, bem-estar psicológico e bem-estar subjetivo

Holland, K. D., \& Holahan, C. K. (2003). The relation of social support and coping to positive adaptation to breast cancer. Psychology and Health, 18(1), 15-29. doi: 10.1080/0887044031000080656

Hu, L.T., \& Bentler, P.M. (1999). Cutoff criteria for fit indexes in covariance structure analysis: conventional criteria versus new alternatives. Structural Equation Modeling, 6, 1-55. doi: 10.1080/10705519909540118

Keyes, C. L. M., Shmotkin, D., \& Ryff, C. D. (2002). Optimizing well-being: the empirical encounter of two traditions. Journal of Personality and Social Psychology, 82(6), 1007-1022. doi: 10.1037/00223514.82.6.1007

John, O. P., \& Gross, J. J. (2004). Healthy and unhealthy emotion regulation: Personality processes, individual differences, and life span development. Journal of Personality, 72(6), 1301-1333. DOI: 10.1111/j.1467-6494.2004.00298.x

Koole, S. L. (2009). The psychology of emotion regulation: an integrative review.Cognition \& Emotion, 23, 4-41. doi: 10.1080/02699930802619031

Lazarus, R. S. (1991). Emotion and adaptation. New York: Oxford University Press.

Livingstone K. M., \& Srivastava, S. (2012). Up regulating positive emotions in everyday life: strategies, individual differences, and associations with positive emotion and well-being. Journal of Research in Personality, 46(5), 504-516. doi: 10.1016/j.jrp.2012.05.009

Lopes, P. N., Salovey, P., Côté, S., \& Beers, M. (2005). Emotion regulation abilities and the quality of social interaction. Emotion, $5,113-118$. doi: 10.1037/1528-3542.5.1.113

Machado, W. L., \& Bandeira, D. R. (2012). Bem-estar psicológico: definição, avaliação e principais correlatos. Estudos de Psicologia, 29(4), 587-595. doi: 10.1590/S0103-166X2012000400013

Machado, W. L., Pawlowski, J., \& Bandeira, D. R. (2013). Validação da Psychological Well-Being Scale em uma amostra de estudantes universitários. Avaliação Psicológica, 12(2), 263-272. Recuperado de http://pepsic.bvsalud.org/scielo.php?script=sci_arttext\&pid=S1677$-04712013000200017 \&$ lng=pt\&tlng=pt.

Marôco, J. (2010). Análise de equações estruturais: fundamentos teóricos, software \& aplicações. Pêro Pinheiro: ReportNumber.

Mauss, I. B., Tamir, M., Anderson, C. L., \& Savino, N. S. (2011). Can seeking happiness make people happy? Paradoxical effects of valuing happiness. Emotion, 11(4), 807-815. doi: 10.1037/a0022010

McMahan, E. A., \& Estes, D. (2011). Measuring lay conceptions of well-being: the beliefs about well-being scale. Journal of Happiness Studies, 12(2), 267-287. doi: 10.1007/s10902-010-9194-x

McMahan, E.A., \& Renken, M. D. (2011) Eudaimonic conceptions of well-being, meaning in life, and self-reported well-being: Initial test of a mediational model. Personality and Individual Differences, 51(5), 589-594. doi: 10.1016/j.paid.2011.05.020

Mendonça, H., Ferreira, M. C., Porto, J., \& Zanini, D. S. (2012). Saúde, qualidade de vida e bem-estar: limites e interfaces teórico-metodológicas. In Saúde e bem-estar no trabalho: dimensões individuais e culturais (pp. 11-34). São Paulo: Casa do Psicólogo.

Nelis, D., Quoidbach, J., Hansenne, M., \& Mikolajczak, M. (2011). Measuring individual differences in emotion regulation: The emotion regulation profile-revised (ERP-R). Psychologica Belgica, 51, 49-91. doi: $10.5334 / \mathrm{pb}-51-1-49$
Nyklíček, I., Denollet, J., \& Vingerhoets, A. (2011) Emotion regulation: Conceptual an clinical issues. Nova lorque: Springer.

Preacher, K. J., \& Hayes, A. F. (2004). SPSS and SAS procedures for estimating indirect effects in simple mediation models. Behavior Research Methods, Instruments, \& Computers, 36(4), 717-731. doi:10.3758/BF03206553

Queroz, N. C., \& Neri, A. L (2005). Bem-estar psicológico e inteligência emocional entre homens e mulheres na meia idade e na velhice. Psicologia: Reflexão e Crítica, 18(2), 292-299. doi: 10.1590/S010279722005000200018

Quoidbach, J., \& Hansenne, M. (2009). The impact of trait emotional intelligence on nursing team performance and cohesiveness. Journal of Professional Nursing, 25(1), 23-29. doi: 10.1016/j.profnurs.2007.12.002

Quoidbach, J., Berry, E. V., Hansenne, M., \& Mikolajczak, M. (2010). Positive emotion regulation and well-being: comparing the impact of eight savoring and dampening strategies. Personality and Individual Differences, 49(5), 368-373. doi: 10.1016/j.paid.2010.03.048

Ryan, R. M., \& Deci, E. L. (2001). On happiness and human potentials: a review of research on hedonic and eudaimonic well-being. Annual Review of Psychology, 52, 141-166. doi: 10.1146/annurev. psych.52.1.141

Ryff, C.D. (1989). Happiness is everything, or is it? Explorations on the meaning of psychological well-being. Journal of Personality and Social Psychology, 57, 1069-1081. doi: 10.1037/0022-3514.57.6.1069

Ryff, C. D., \& Keyes, C. L. (1995). The structure of psychological wellbeing revisited. Journal of Personality and Social Psychology, 69(4), 719-727. doi: 10.1037/0022-3514.69.4.719

Ryff, C. D., \& Singer B. H. (2008). Know thyself and become what you are: a eudaimonic approach to psychological well-being. Journal of Happiness Studies, 9, 13-39. doi: 10.1007/s10902-006-9019-0

Ryff, C. D., Keyes, C. L. M., \& Hughes, D. L. (2003). Status Inequalities, perceived discrimination, and eudaimonic well-being: do the challenges of minority life hone purpose and growth? Journal of Health and Social Behavior, 44(3),275-291. Recuperado de http://dx.doi. org/10.1590/S0103-166X2012000400013

Segrin, C.; \& Taylor, M. (2007). Positive interpersonal relationships mediate the association between social skills and psychological well-being. Personality and Individual Differences, 4, 637-646.

Siqueira, M. M. M., \& Padovam, V. A. R (2008). Bases teóricas de bem-estar subjetivo, bem-estar psicológico e bem-estar no trabalho. Psicologia: Teoria e Pesquisa, 24(2), 201-209. doi: 10.1590/S010237722008000200010.

Sobel, M. E. (1982). Asymptotic confidence intervals for indirect effects in structural equation models. In Leinhardt, S. (Org.), Sociological Methodology (pp. 290-312). Washington DC: American Sociological Association.

Tamir, M. (2011). The maturing field of emotion regulation. Emotion Review, 3, 3-7. doi: 10.1177/1754073910388685

Tamir, M., Mitchell, C., \& Gross, J. J. (2008). Hedonic and instrumental motives in anger regulation. Psychological Science, 19, 324-328. doi: 10.1111/j.1467-9280.2008.02088.x

Warr, P. (2007). Work, happiness, and unhappiness. Mahwah: Lawrence Erlbaum Associates, Inc. 
Vitor Santos Santana, Mestre em Psicologia Social e do Trabalho pela Universidade Federal da Bahia (UFBA). Endereço para correspondência: Rua dos Bandeirantes no 872, Matatu Salvador/Bahia/Brasil. CEP: 40.260-000. Telefone: (71) 99171-3251. E-mail: vitor_santana23@hotmail.com

Sônia Maria Guedes Gondim, Doutora em Psicologia Social e da Personalidade pela Universidade Federal do Rio de Janeiro (UFRJ),

Pós-doutorado pela Universidad Complutense de Madrid, é Professora Associada Nível IV no Instituto de Psicologia Universidade Federal da Bahia (UFBA). E-mail: sggondim@gmail.com 